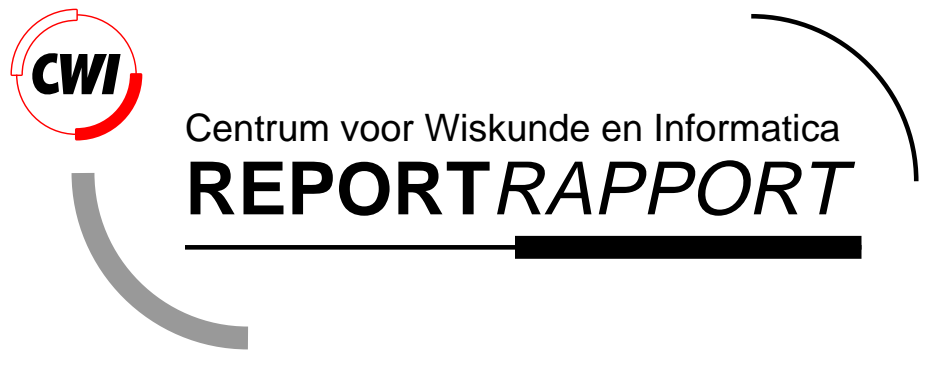

A Conserving Discretisation for a Stefan Problem with an Interface Reaction at the Free Boundary

C. Vuik, A.Segal, F.J. Vermolen

Modelling, Analysis and Simulation (MAS)

MAS-R9904 March 1999 
Report MAS-R9904

ISSN 1386-3703

CWI

P.O. Box 94079

1090 GB Amsterdam

The Netherlands

CWI is the National Research Institute for Mathematics and Computer Science. CWI is part of the Stichting Mathematisch Centrum (SMC), the Dutch foundation for promotion of mathematics and computer science and their applications.

SMC is sponsored by the Netherlands Organization for Scientific Research (NWO). CWI is a member of ERCIM, the European Research Consortium for Informatics and Mathematics.

Copyright $(0$ Stichting Mathematisch Centrum P.O. Box 94079, 1090 GB Amsterdam (NL) Kruislaan 413, 1098 SJ Amsterdam (NL) Telephone +3120 5929333 Telefax +3120 5924199 


\title{
A Conserving Discretization for a Stefan Problem with an Interface Reaction at the Free Boundary
}

\author{
Kees Vuik, Guus Segal \\ Faculty of Technical Mathematics and Informatics \\ Delft University of Technology \\ P.O.Box 5031, 2600 GA Delft, The Netherlands \\ Fred Vermolen \\ $C W I$ \\ P.O.Box 94079, 1090 GB Amsterdam, The Netherlands
}

\begin{abstract}
The dissolution of an $\mathrm{Al}_{2} \mathrm{Cu}$ particle is considered. A characteristic property is that initially the particle has a non-smooth boundary. Furthermore the dissolution may be controlled by an interface reaction. The mathematical model of this dissolution process contains a description of the particle interface, of which the position varies in time. Such a model is called a Stefan problem. We use the finite element method to solve this problem numerically. The displacement of the free boundary is computed by a method based on the balance of atoms. This method leads to good results, also for non-smooth boundaries. Some numerical experiments are given for the dissolution of an $\mathrm{Al}_{2} \mathrm{Cu}$ particle in an $\mathrm{Al}-\mathrm{Cu}$ alloy, with a varying rate of the interface reaction.
\end{abstract}

1991 Mathematics Subject Classification: 35A35, 35R35, 65M06, 80A22

Keywords and Phrases: Stefan problem, moving finite elements, conserving discretisation, particle dissolution, interface reaction Note: Research carried out under the Welgel project. The paper has been submitted to the Journal Computing and Visualisation in Science.

\section{INTRODUCTION}

Heat treatment of metals is often necessary to optimize their mechanical properties. During the heat treatment, the metallurgical state of the material changes. This change can either involve the phases being present or the morphology of the various phases. One of these processes, which is both of large industrial and scientific interest and amenable to modeling, is the dissolution of second phase particles in a matrix with a uniform initial composition.

To describe this particle dissolution in rigid media several physical models have been developed, incorporating the effects of long-distance diffusion [9], [2], [6] and non-equilibrium conditions at the interface [3], [1]. The long-distance diffusion models imply that the processes at the interface between particle and matrix proceed infinitely fast. Therefore, these models provide an upper bound for the dissolution rate.

Nolfi's model [3] did not include the interface migration, but as far as we know, it is the first model which incorporated non-equilibrium conditions at the interface. In the Nolfi model nonequilibrium conditions at the interface were incorporated by the introduction of a Robbins 
condition, which relates the concentration gradient with the concentration at the interface. The semi-analytical solution consists of an infinite series solution for the concentration profile. Their method, however, is only accurate in the early stages of the dissolution process.

Aaron and Kotler [1] incorporated the non-equilibrium conditions at the interface too. However their approach is only applicable for those situations in which the inter-particle distance is sufficiently large, i.e. the diffusion fields do not impinge. They transformed the Robbins problem of Nolfi into a Dirichlet problem, in which the concentration is fixed at all stages of the dissolution process. Combining Whelan's [9] analytical approach for the interface velocity as a function of the annealing time, with a relation between the interface concentration and the interface position, they evaluated the interface position using a Picard-type iteration method. In their model both the interface position and the interface concentration were taken momentarily stationary during the evaluation of the interface position as a function of time.

The effects of interfacial reactions on the rate of the dissolution of spherical particles in both infinite and finite media was examined by Vermolen and Van der Zwaag numerically [7]. Using a finite difference method it was shown, that interfacial reactions can have a significant effect on the dissolution rate and hence on the concentration profiles in the matrix during particle dissolution.

All solution methods presented in the references given above are only applicable to onedimensional problems. In [5] a method is described by Segal, Vuik and Vermolen to compute a numerical solution of a two-dimensional Stefan problem with a conserving discretization of the free boundary. This method is applied for an infinite rate of the interface reaction.

In this paper we generalize the method presented in [5] to a Stefan problem with a finite rate of reaction at the interface. The boundary conditions at the free boundary are derived in Section 2 and compared with the literature. In Section 3 the numerical method presented in [5] is summarized. The numerical discretization of the interface reaction is investigated in Section 4. In Section 5 some results are shown.

\section{A MOdEL FOR Dissolution With AN INTERFACE REACTION}

Consider an $\mathrm{Al}_{2} \mathrm{Cu}$ particle in an $\mathrm{Al}-\mathrm{Cu}$ alloy at a given temperature. The initial concentration of $\mathrm{Al}_{2} \mathrm{Cu}$ in the Aluminum phase is equal to $c_{0}\left(\mathrm{~mol} / \mathrm{m}^{3}\right)$, whereas $c_{\text {part }}$ denotes the concentration of $\mathrm{Al}_{2} \mathrm{Cu}$ in the particle. When the temperature is increased, dissolution of the $\mathrm{Al}_{2} \mathrm{Cu}$ particle sets in. The equilibrium $\mathrm{Al}_{2} \mathrm{Cu}$ concentration in the alloy is $\mathrm{c}_{\text {sol }}$ $\left(c_{\text {part }}>c_{\text {sol }}>c_{0}\right)$.

To describe the mathematical model we use the geometry as given in Figure 1. The domain filled with Aluminum is denoted by $\Omega(t)$. The boundary of this domain consists of the interface $S(t)$ and the outer boundaries $\Gamma_{i}, \quad i \in\{1,2,3,4\}$. The outer boundaries are fixed in time, except the intersections of $\Gamma_{1}$ and $\Gamma_{4}$ with $S(t)$. In the Aluminum-rich phase $\Omega(t)$, the $C u$ concentration $c(x, y, t)$ satisfies the (linear) diffusion equation

$$
\frac{\partial c}{\partial t}=\mathbb{D} \Delta c, \quad(x, y) \in \Omega(t), \quad t \in(0, T]
$$




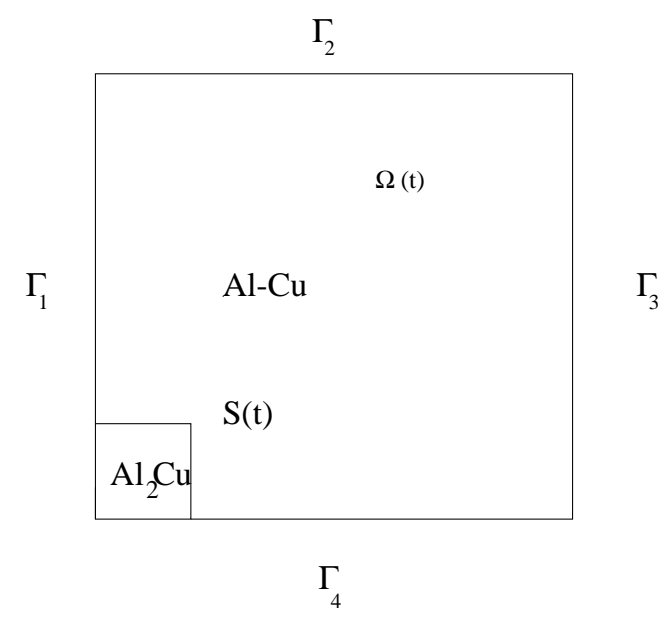

Figure 1: Geometry of an $\mathrm{Al}_{2} \mathrm{Cu}$ particle in Aluminum

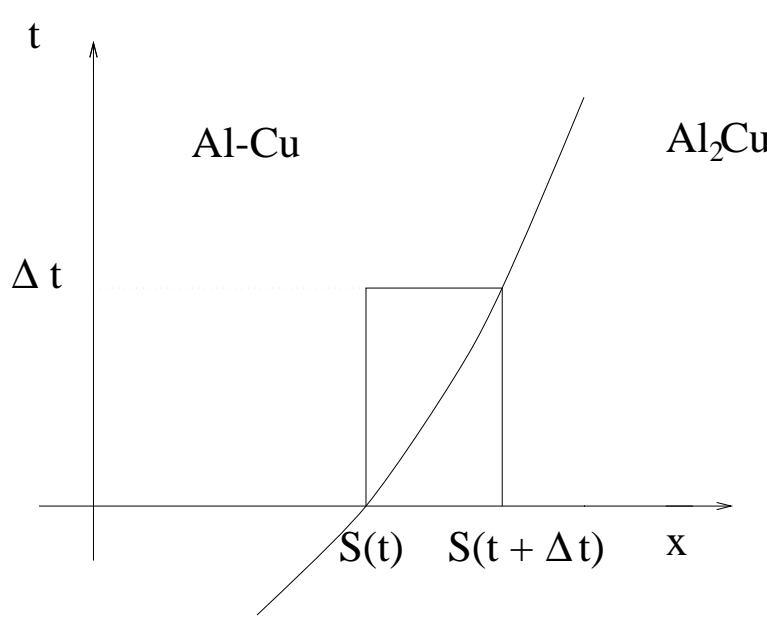

Figure 2: The control volume

The diffusion coefficient $\mathbb{D}\left(\mathrm{m}^{2} / \mathrm{s}\right)$ is supposed to be independent of concentration. As the initial condition we use

$$
c(x, y, 0)=c_{0}(x, y), \quad(x, y) \in \Omega(0),
$$

where $\Omega(0)$ is prescribed. We assume no flux of $C u$ through the outer boundaries, so

$$
\frac{\partial c}{\partial \mathbf{n}}(x, y, t)=0, \quad(x, y) \in \Gamma_{i}, \quad i \in\{1,2,3,4\}, \quad t \in[0, T] .
$$

To determine the position of the interface two conditions are necessary. To derive these conditions for a spatially three dimensional problem, we consider a small part of the interface. Suppose that the interface is smooth, which means that it can locally be described by differentiable functions. For a small time step $\Delta t$ the interface moves in the direction perpendicular to the interface. The $x$-axis is chosen along the normal. With this choice the position of the interface is locally described by the relation $x=S(t)$. We consider a control volume of width $\Delta y$ and $\Delta z$. The intersection of the control volume with the surface $y=0, z=0$ is shown in Figure 2. The balance of $C u$ atoms leads to the following equation (Stefan condition):

$$
(S(t+\Delta t)-S(t)) \Delta y \Delta z \cdot c_{p a r t}=\mathbb{D} \frac{\partial c}{\partial x} \Delta y \Delta z \Delta t+(S(t+\Delta t)-S(t)) \Delta y \Delta z \cdot c_{S},
$$

where $c_{S}$ is the limit of the concentration in $\Omega$ in the neighborhood of the interface. The lefthand side of (2.4) is equal to the amount of atoms transferred from the particle to the alloy. Assuming a first order reaction at the interface the second equation is (Robbins condition):

$$
K\left(c_{s o l}-c_{S}\right) \Delta y \Delta z \Delta t=\mathbb{D} \frac{\partial c}{\partial x} \Delta y \Delta z \Delta t+(S(t+\Delta t)-S(t)) \Delta y \Delta z \cdot c_{S},
$$

where $K(\mathrm{~m} / \mathrm{s})$ is a measure of the rate of the interface reaction. For $K$ large the problem is diffusion controlled, whereas for $K$ small the problem is reaction controlled. Dividing (2.4) and (2.5) by $\Delta y \Delta z \Delta t$ and taking the limit $\Delta t \rightarrow 0$ one obtains

$$
c_{\text {part }} v_{n}(x, y, t)=\mathbb{D} \frac{\partial c}{\partial \mathbf{n}}(x, y, t)+c_{S} v_{n}(x, y, t), \quad(x, y) \in S(t), \quad t \in(0, T],
$$




$$
K\left(c_{s o l}-c_{S}\right)=\mathbb{D} \frac{\partial c}{\partial \mathbf{n}}(x, y, t)+c_{S} v_{n}(x, y, t), \quad(x, y) \in S(t), \quad t \in(0, T]
$$

where $\mathbf{n}$ is the unit normal vector on the interface pointing outward with respect to $\Omega(t)$ and $v_{n}$ is the normal velocity of the interface.

In the references [3], [7] and [8] comparable boundary conditions are used. In [3] and [7] the final term in (2.7) is not taken into account. This approximation is reasonable because in their applications $c_{s o l} \ll c_{\text {part }}$, which implies that the neglected term is small.

\section{A CONSERVing COMputation OF the FREE BOUndaRy FOR INFinite RATE OF REAC- TION}

In the literature (see [5] for a short overview) one can find various numerical methods to solve Stefan problems. These methods can be distinguished in the following categories: fronttracking, front-fixing and fixed-domain methods. We shall restrict ourselves to front-tracking since it allows first order reaction at the interface.

The algorithm for an infinite rate of reaction can be described as follows. In each time-step we solve the (ALE) convection-diffusion equation

$$
\frac{D c}{D t}-\mathbb{D} \Delta c-\mathbf{u}_{m e s h} \cdot \nabla c=0,
$$

with $\frac{D c}{D t}$ the so-called material derivative and $\mathbf{u}_{m e s h}=\frac{\mathbf{x}(t+\Delta t)-\mathbf{x}(t)}{\Delta t}$ the mesh velocity. After that, the boundary is updated according to

$$
\mathbf{x}(t+\Delta t)=\mathbf{x}(\mathbf{t})+v_{n} \Delta t \mathbf{n}=\mathbf{x}(\mathbf{t})+\frac{\mathbb{D}}{c_{\text {part }}-c_{\text {sol }}} \frac{\partial c}{\partial \mathbf{n}} \Delta t \mathbf{n} .
$$

The straight-forward way to update the free boundary is to compute the gradient of the concentration in the elements connected to the free boundary (normal_velocity method). Using an averaging procedure for the gradient as well as an averaging procedure to compute the normals in the vertices of the boundary, equation (3.2) can be evaluated.

However, in case of sharp corners this may lead to a strange behavior as is shown in Figure 3. In order to get rid of this phenomenon we have developed a new algorithm (Stefan) based on the integral representation of the Stefan boundary condition. This implies that the area of the particle that has been dissolved is equal to the amount of diffused material. The flux through the element $\left(x_{i-1}, x_{i}\right)$ is approximately equal to:

$$
\mathbb{D} \frac{\partial c}{\partial \mathbf{n}}\left(x_{i-\frac{1}{2}}\right) l_{i} \Delta t
$$

with $l_{i}$ the length of the line element $\left(x_{i-1}, x_{i}\right)$. Hence the amount of diffused material through the boundary $\left(x_{i-\frac{1}{2}}, x_{i+\frac{1}{2}}\right)$ is equal to

$$
\frac{\Delta t}{2}\left(\mathbb{D} \frac{\partial c}{\partial \mathbf{n}}\left(x_{i-\frac{1}{2}}\right) l_{i}+\mathbb{D} \frac{\partial c}{\partial \mathbf{n}}\left(x_{i+\frac{1}{2}}\right) l_{i+1}\right)
$$

The amount $M$ of material dissolved, is approximately equal to $\left(c_{\text {part }}-c_{\text {sol }}\right) O$, where $O$ is the area defined in Figure 5. Due to the balance of atoms $M$ must be equal to the amount 


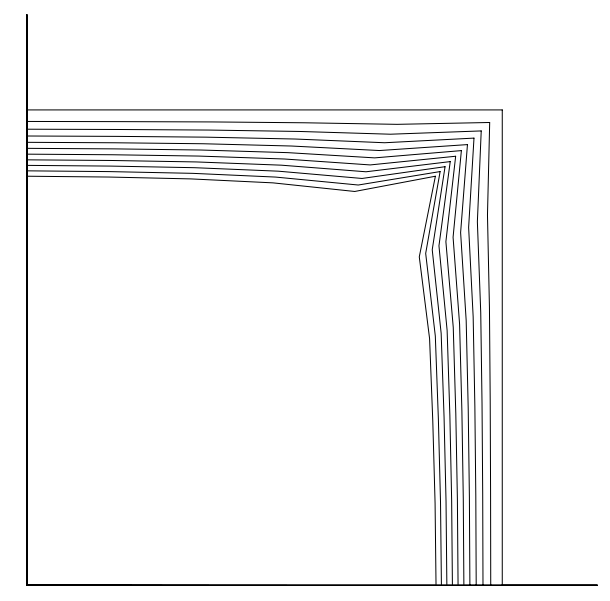

Figure 3: Position of free boundary at first 10 time-steps using the normal_velocity method for infinite rate of reaction

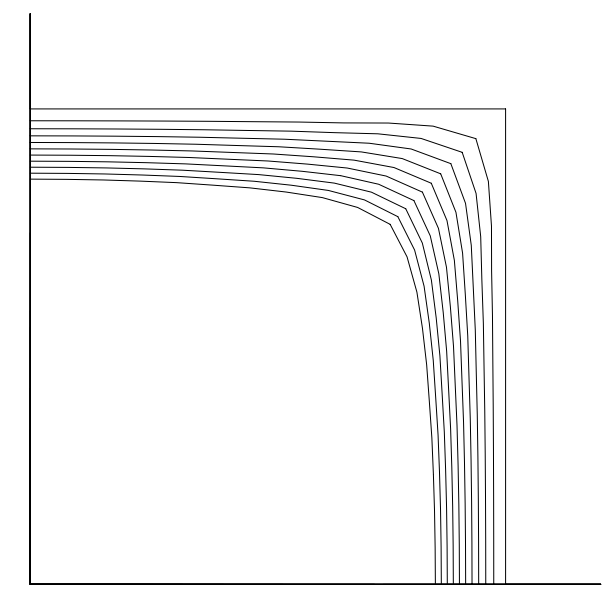

Figure 4: Position of free boundary at first 10 time-steps using the Stefan method for infinite rate of reaction

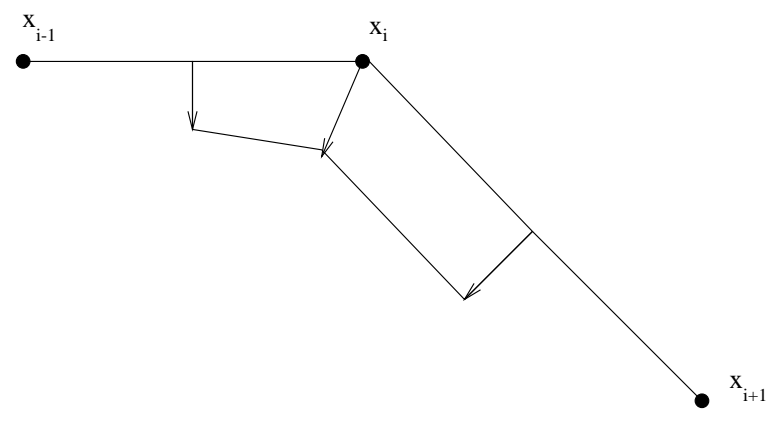

Figure 5: Area occupied by the region defined by the displacement of the vertex

of diffused material given in Equation (3.4). Consider two adjacent line elements $\left(x_{i-1}, x_{i}\right)$, and $\left(x_{i}, x_{i+1}\right)$, with length $l_{i}$ and $l_{i+1}$ respectively (Figure 5$)$. The mid-side points of these elements are denoted by $x_{i-\frac{1}{2}}$ and $x_{i+\frac{1}{2}}$. Let the from formula (3.2) computed displacement in the mid-side points, be equal to $\delta x_{i-\frac{1}{2}}$ and $\delta x_{i+\frac{1}{2}}$. The new position of vertex $x_{i}$ is denoted by $\hat{\mathbf{x}}_{i}$. The length of the displacement is given as $\Delta x_{i}=\left\|\hat{\mathbf{x}}_{i}-\mathbf{x}_{i}\right\|$. Once the displacement in the vertices is computed, also the displacements in the mid-side points change. In order to get both a local and global equilibrium in the amount of dissolved material, it is necessary, that the new area is equal to

$$
\frac{1}{2} l_{i} \delta x_{i-\frac{1}{2}}+\frac{1}{2} l_{i+1} \delta x_{i+\frac{1}{2}} \cdot
$$

The area $O$ depends on $\Delta x_{i}, \Delta x_{i-\frac{1}{2}}$ and $\Delta x_{i+\frac{1}{2}}$, where $\Delta x_{i-\frac{1}{2}}$ is the adapted length of the displacement in $x_{i-\frac{1}{2}}$. Since $\Delta x_{i-\frac{1}{2}}$ and $\Delta x_{i+\frac{1}{2}}$ depend on $\Delta x_{i-1}, \Delta x_{i}$ and $\Delta x_{i+1}$ the relation is non-linear.

To solve this non-linear system we had to use an under-relaxation parameter. Choosing this parameter equal to 0.5 gave a fast convergence. The results of the Stefan algorithm are shown in Figure 4. The results in Figure 4 are more reliable than those in Figure 3 since from 
physical point of view we expect a large diffusion of the atoms at the angular free boundary point. This gives locally larger free boundary velocities. For more details we refer to [5].

\section{The DiscRetization For a Finite RATE OF REACtion}

The method summarized in Section 3 has been developed for a Stefan problem with an infinite fast interface reaction, so the concentration at the free boundary is equal to $c_{\text {sol }}$. To generalize this method to a finite rate of reaction one has to discretize the boundary conditions (2.6) and (2.7). Both conditions contain the normal velocity of the interface. In our implementation we use the Robbins condition (2.7) to solve the diffusion equation and the Stefan condition (2.6) to adapt the free boundary. In order to get rid of the normal velocity in (2.7) equation (2.6) is substituted into (2.7):

$$
K\left(c_{\text {sol }}-c_{S}\right)=\mathbb{D} \frac{c_{\text {part }}}{c_{\text {part }}-c_{S}} \frac{\partial c}{\partial \mathbf{n}}(x, y, t), \quad(x, y) \in S(t), \quad t \in(0, T] .
$$

This equation is linearized by taking $c_{S}$ in the right-hand side of (4.1) at the old time level.

Experiments with boundary condition (4.1) in combination with the Stefan algorithm to adapt the free boundary, showed inaccuracies for a finite rate of reaction. The computed shapes of the free boundary resemble the shapes shown in Figure 3. The approximation of the normal gradient of $c$ used in (3.3) may be the cause of these instabilities. To avoid this approximation, (2.7) is subtracted from (2.6) which yields:

$$
v_{n}(x, y, t)=K \frac{c_{\text {sol }}-c_{S}}{c_{\text {part }}}, \quad(x, y) \in S(t), \quad t \in(0, T] .
$$

Note that for $K \rightarrow \infty, v_{n}$ is bounded since $c_{S} \rightarrow c_{s o l}$. However when the difference between $c_{S}$ and $c_{s o l}$ is very small it is not practical to use (4.2) because cancellation can occur.

To adapt the free boundary the Stefan algorithm (3.5) combined with (4.2) is used. In this algorithm the velocities are used in the mid-side points of the element boundaries, whereas the concentrations are given in the vertices of the elements. Therefore $c_{S}\left(x_{i-\frac{1}{2}}\right)$ is approximated by averaging the $c_{S}$ in the vertices and substitute this value into equation (4.2).

\section{NumERICAL EXPERIMENTS}

An algorithm has been developed to investigate the dissolution kinetics for a two-dimensional case with a first order reaction at the interface. This algorithm has been implemented in our finite element code SEPRAN [4]. As an example we consider the dissolution of a needle shaped particle in a bar. Due to the symmetry of this two-dimensional problem, we restrict the simulation to one quarter of the real geometry. First we investigate the influence of the rate of the interface reaction $(K)$ on the shape of the dissolving particle. Thereafter we compare the influence of the extra terms used in (2.6) and (2.7). In all our examples we have chosen the following parameters:

$\begin{array}{lrl}\text { diffusion coefficient } & \mathbb{D}=0.04858, \\ \text { concentration in the particle } & c_{\text {part }}=54, \\ \text { initial concentration } & c_{0}=0.0011 .\end{array}$




\subsection{The influence of the interface reaction}

We consider a square dissolving in a square for $c_{s o l}=3.88$ and various choices of $K$. In Figure 6 we present the results as given in ([5], Fig. 21) for the Dirichlet condition at the interface. The next figures contain the results for $K=1000,10$, and 0.1 . For $K$ large we expect that

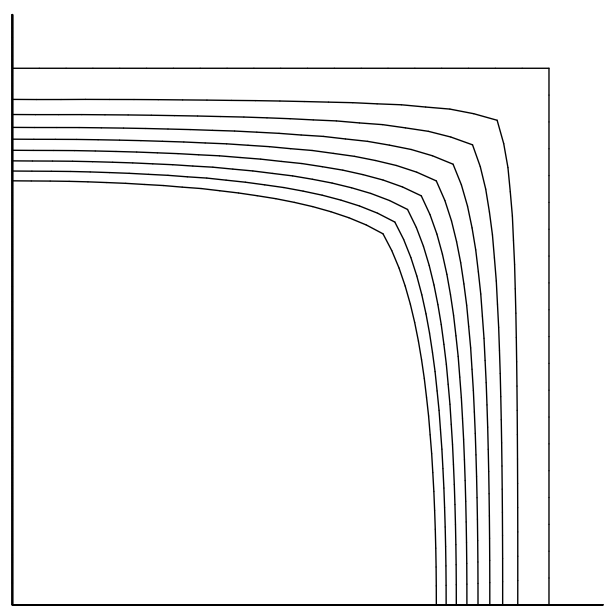

Figure 6: Free boundary of a bar dissolving in a bar using a Dirichlet boundary condition

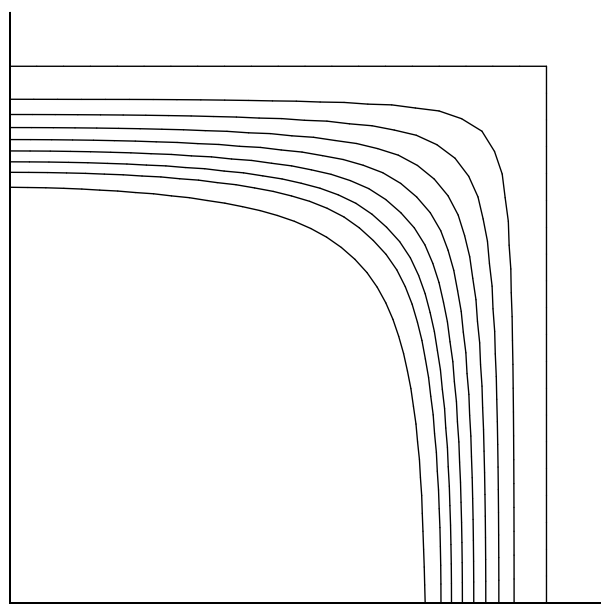

Figure 7: Free boundary of a bar dissolving in a bar with $K=1000$

the solution converges to the solution of the Dirichlet problem. However comparing Figure 6 and 7 it appears that the latter figure shows a more rounded profile of the particle. The reason for this is that in Figure 6 the fluxes are approximated by the normal derivative of the concentration in the mid-side points. In the vicinity of the corner these derivatives appear to be underestimated. For $K=1000$ the fluxes are approximated by equation (4.2), which is based only on the concentration. When the grid is refined we observe that the Dirichlet solution converges to the solution as given in Figure 7. So we expect that this solution is more accurate than the solution of the Dirichlet problem for the same grid size.

For small values of $K$ the evolution of the position of the interface is completely determined by the rate of the interface reaction. Therefore one expects that the particle remains squarelike. This is in accordance with the results as given in Figure 8 and 9. Also the velocity of the interface decreases when $K$ decreases.

\subsection{The influence of the term $c_{S} v_{n}$}

In the derivation of the model we have already noted that in some references the term $c_{S} v_{n}$ is deleted from equation (2.7). For the problem as considered in Section 5.1 we have compared the solution with and without this term and it appears that its influence is negligible. On the other hand when $c_{s o l}$ is closer to $c_{\text {part }}$ the differences may be large. Therefore we consider an academic problem where $c_{s o l}$ is 10 times as large: $c_{s o l}=38.8$ and take $K=0.1$. The results of the correct boundary conditions are given in Figure 10. Since $c_{\text {sol }}$ is much larger the velocity of the interface is much higher. Therefore the time-steps used in these problems are equal to the time-steps of the previous problem divided by 10. The results given in Figure 11 are obtained when the term $c_{S} v_{n}$ is deleted from equation (2.7). There are considerable differences between both results. Neglecting $c_{S} v_{n}$ leads to an overestimate of the position of 


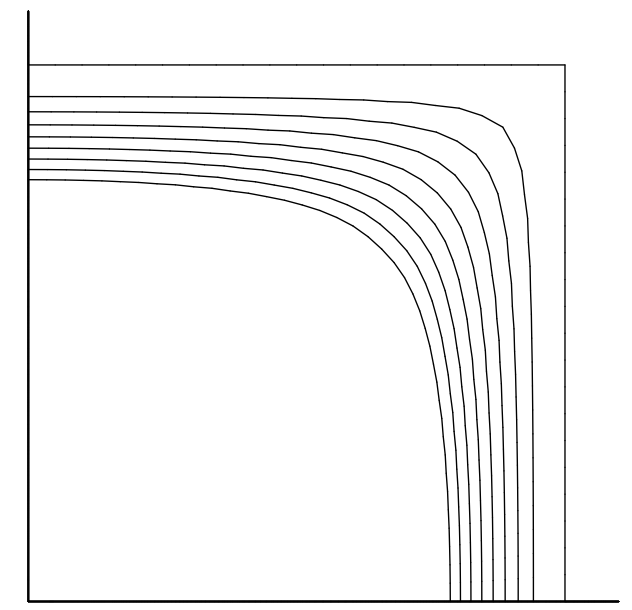

Figure 8: Free boundary of a bar dissolving in a bar with $K=10$

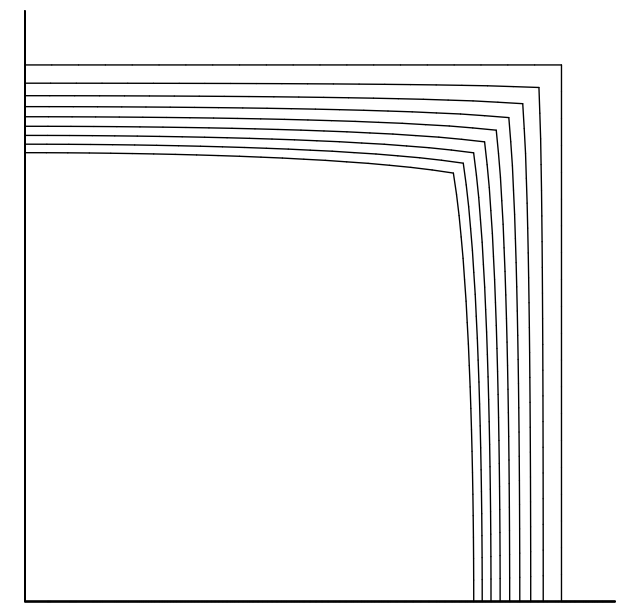

Figure 9: Free boundary of a bar dissolving in a bar with $K=0.1$

the free boundary.

\section{Conclusions}

Particle dissolution in binary alloys is investigated by numerical techniques. The mathematical model used is that of a free boundary problem of Stefan type with a parameter which allows to switch between "diffusion controlled" and "interface controlled" migration. The problem is solved by a two-dimensional finite element method. It has been shown that this approach leads to an accurate solution of the problem.

It appears that the solution of the model with a fast reaction on the interface leads to better results than the model with a Dirichlet condition at the interface. Therefore we recommend to use the numerical solution of the model with an interface reaction with $K$ sufficiently large to approximate the solution of the model with the Dirichlet boundary condition. As expected the influence of the diffusion disappears when the reaction is slow. In such a case the shape of the dissolving particle remains the same during dissolution.

In some references the boundary conditions are only approximately true. It has been shown that this approximation is allowed for the problems considered. However in other applications where $c_{s o l}$ and $c_{\text {part }}$ have the same order of magnitude all terms should be included in the boundary conditions. 


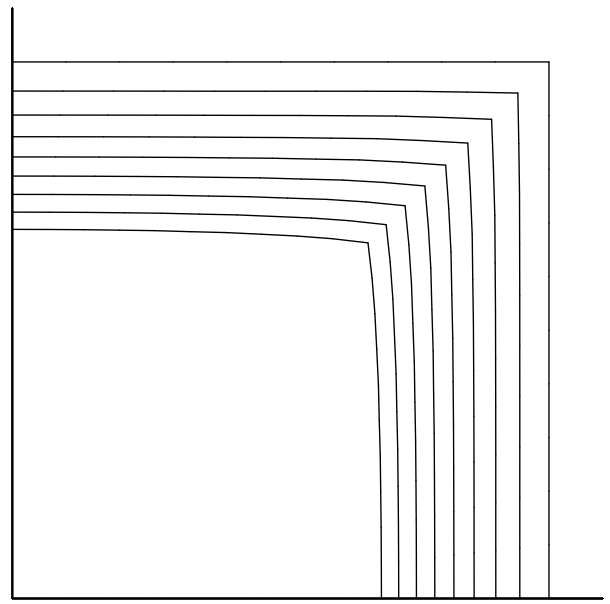

Figure 10: Free boundary of a bar dissolving in a bar with $K=0.1$ and $c_{\text {sol }}=38.8$

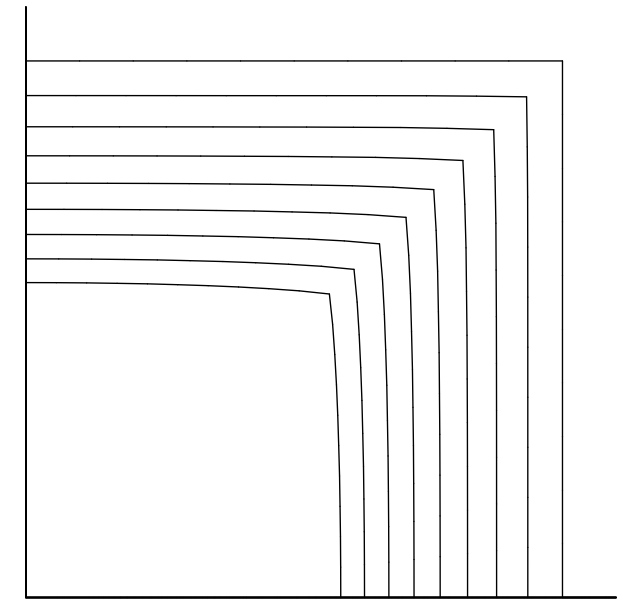

Figure 11: Free boundary of a bar dissolving in a bar with $K=0.1$ and $c_{s o l}=38.8$ without the term $c_{S} v_{n}$ 


\section{References}

1. H.B. Aaron and G.R. Kotler. Second phase dissolution. Metallurgical Transactions, 2:393407, 1971.

2. U.L. Baty, R.A. Tanzilli, and R.W. Heckel. Dissolution kinetics of CuAl2 in an Al-4Cu alloy. Metallurgical Transactions, 1:1651-1656, 1970.

3. F.V. Nolfi Jr., P.G. Shewmon, and J.S. Foster. The dissolution and growth kinetics of spherical precipitates. Transactions of the Metallurgical Society of AIME, 245:1427-1433, 1969.

4. Guus Segal. SEPRAN manuals. Leidschendam, 1984.

5. Guus Segal, Kees Vuik, and Fred Vermolen. A conserving discretization for the free boundary in a two-dimensional Stefan problem. J. Comp. Phys., 141:1-21, 1998.

6. U.H. Tundal and N. Ryum. Dissolution of particles in binary alloys: Part I. computer simulations. Metallurgical Transactions, 23A:433-449, 1992.

7. F.J. Vermolen and S. Van der Zwaag. A numerical model for the dissolution of spherical particles in binary alloys under mixed mode control. Materials Science and Engineering A, 220:140-146, 1996.

8. C. Vuik and C. Cuvelier. Numerical solution of an etching problem. J. Comp. Physics, 59:247-263, 1985.

9. M.J. Whelan. On the kinetics of particle dissolution. Metal Science Journal, 3:95-97, 1969. 